\title{
Hepatocellular Carcinoma: Basic and Transitional Research
}

\author{
Chuan Yin Wei-Fen Xie \\ Department of Gastroenterology, Changzheng Hospital, Second Military Medical University, \\ Shanghai, PR China
}

\section{Key Words}

Cancer stem cells · Differentiation · Hepatocellular carcinoma - Molecular-targeted therapies . Signaling pathways

\begin{abstract}
Background: Hepatocellular carcinoma (HCC) is one of the leading causes of cancer-related deaths worldwide. The outcome of HCC therapy depends on the stage of HCC. Early-stage HCC patients can be cured with radical treatment approaches, whereas no standard treatment regimens can be recommended for patients with advanced disease.

Summary: In-depth basic research into the molecular mechanisms of HCC has contributed to the development of novel therapeutic agents. This article reviews several key classes of novel therapeutic agents that are under development, including molecular-targeted therapies, cancer stem cell (CSC)-based therapy and differentiation therapy.

Key Message: A greater understanding of the molecular pathogenesis of HCC has contributed to the development of novel therapeutic agents. This article reviews several key classes of novel therapeutic agents that are under development, including molecular-targeted therapies, CSC-based therapy and differentiation therapy.

Practical Implications: Molecular-targeted therapies based on signaling pathways involved in hepatocarcinogenesis and progression are being evaluated in several clinical trials. There are three main categories of targeted agents: tyrosine kinase inhibitors (TKIs), monoclonal antibodies and enzyme inhibitors. The best-established agent is sorafenib, a non-specific TKI that is accepted as first-line therapy for specific patients. Other similar agents under investigation include erlotinib, linifanib and brivanib. CSC-based therapies are still in the earlier stages of development and include a neutralizing anti-CD44 antibody, small interfering RNA to suppress epithelial cell adhesion molecular levels, a neutralizing anti-CD13 antibody and a
\end{abstract}


CD13 inhibitor. An important point is that CSC-targeted therapy should be combined with conventional therapies to achieve complete tumor regression. Differentiation therapy is defined as a strategy that induces malignant reversion of tumor cells. Hepatocyte nuclear factor $4 \alpha$ or $1 \alpha$, important transcriptional factors for hepatocyte differentiation and phenotype maintenance, have shown significant antitumor effects by inducing differentiation of both non-CSCs and CSCs in HCC towards a hepatocyte-like phenotype.

(C) 2014 S. Karger AG, Basel

\section{Introduction}

Hepatocellular carcinoma (HCC) is the third leading cause of cancer-related death worldwide. Although over $80 \%$ of HCC cases occur in sub-Saharan Africa and Asian countries, the number of diagnosed cases has also been increasing over the past decade in several Western countries with previously low incidence levels [1].

The outcome of HCC treatment is highly dependent on the stage of the disease at diagnosis. Liver resection and transplantation have been proven as the best modalities for earlystage patients [2]. Local ablation has also been proposed as an alternative for patients at early stages who are not suitable for surgery. HCC patients with intermediate disease may benefit from palliative treatments, such as transarterial chemoembolization (TACE), TACE with doxorubicin-eluting beads and radioembolization [2-4]. However, terminal patients rarely benefit from any available treatments [5]. As most HCC patients present with advanced-stage disease, their prognosis is extremely dismal and highly effective approaches with low toxicity are urgently needed.

\section{Molecular-Targeted Therapies}

Molecular-targeted therapies aim to only interfere with specific molecules necessary for tumor growth and progression. Although traditional chemotherapies often kill rapidly dividing cells in the body by interfering with cell division, they usually cause severe side effects such as myelotoxicity. In general, systemic chemotherapy is not particularly effective in HCC patients. A primary goal of molecular-targeted therapies is to target cancer cells with greater precision and fewer side effects.

It is well known that carcinogenesis is a complex multistep process with multiple altered signaling cascades. Several signaling pathways have been implicated in hepatocarcinogenesis, including vascular endothelial growth factor (VEGF) signaling, epidermal growth factor (EGF) signaling, RAS/mitogen-activated protein kinase (MAPK) signaling, the PI3K/PTEN/AKT/ mTOR pathway, the HGF/c-MET pathway, insulin-like growth factor receptor (IGFR) signaling and the WNT/ $\beta$-catenin pathway. Thus, the components of these pathways constitute the molecular targets for cancer therapy.

Targeted agents mainly include three types: tyrosine kinase inhibitors (TKIs), monoclonal antibodies (mAbs) and enzyme inhibitors. Most TKIs can influence cellular proliferation and/or angiogenesis by targeting multiple receptors such as VEGFR, PDGFR, EGFR, FGFR and IGFR. The best-characterized clinical application of the molecular-targeted therapy for advanced HCC is the use of sorafenib, a non-specific TKI that targets both cell surface tyrosine kinase receptors and downstream intracellular serine/threonine kinases in the RAS/MAPK cascade [6]. It has been accepted as the first-line therapy for patients who will not benefit from resection, transplantation, ablation or TACE and still have well-preserved liver function (Child-Pugh class A) [2, 3, 5]. Furthermore, various trials of sorafenib-based combi- 
nations are currently ongoing in the clinic, including sorafenib combined with chemotherapeutics or radiation, as well as other targeted agents; these have widely exhibited promising results [7]. Although sorafenib-based therapies are an important advance in HCC therapy, a number of questions remain unresolved. Recent studies reported that multiple lung metastases were frequently observed among responders to sorafenib but were less common among non-responders, which was consistent with the fact that antiangiogenesis drugs may cause recurrence of tumor growth or increased local invasion and distant metastasis in certain situations [8]. Moreover, research by Zhang et al. [9] revealed that sorafenib pretreatment accelerated tumor growth, promoted lung metastasis and decreased survival in mice. These effects could be attributable to a direct inhibitory effect of sorafenib on the proliferation and activation of natural killer cells. Zhang et al. [8] also reported that sorafenib could promote the invasiveness of HCC cells through downregulating the expression of HIV-1 Tat interactive protein 2 (HTATIP2) via suppressing Jun-activated kinase and signal transducer and activator of transcription 3 (STAT3) signaling. They also found that the sorafenib-promoted invasiveness occurred only in those HCC cell lines with higher levels of HTATIP2. Interestingly, the proinvasive effects were limited to relatively low dosages of sorafenib treatment or when the therapy was discontinued. In contrast, at higher and continuous dosages sorafenib inhibited the growth of most tumor cells and the proinvasive effect was eliminated. These results suggest that patients with lower expression of HTATIP2 in the tumor may present as better candidates for sorafenib treatment [8]. Collectively, the benefit of sorafenib-based therapies still remains elusive. Further efforts should be made to select the subpopulation of HCC patients who may benefit from sorafenib treatment.

Stimulated by the success of sorafenib, several TKIs are currently being evaluated in multiple clinical trials, including erlotinib (a TKI of EGFR), linifanib (a TKI of VEGFR and PDGFR), brivanib (a TKI of FGFR, VEGFR and PDGFR), OSI-906 (a TKI of IGF-1R), AZD6244/ selumetinib (a MEK inhibitor) as well as ARQ 197 and foretinib (c-MET inhibitors). In addition, four mTOR inhibitors (TKIs of MTORC1), including temsirolimus, everolimus, rapamycin and AZD8055, are also under investigation.

mAbs, the second most commonly used cancer therapy, usually target cell surface receptors and/or secreted growth factors. Compared with TKIs, the advantages of mAbs are less frequent dosing because of their longer half-life and increased specificity resulting in decreased toxicity [10]. One representative molecular-targeted drug, trastuzumab (Herceptin), is a mAb that interferes with the HER2/neu receptor. Women with HER2/neupositive disease who received trastuzumab treatment had a $44 \%$ reduction in the risk of death compared with women with HER2/neu-negative disease [11].

Bevacizumab, a recombinant humanized $m A b$ against VEGF-A, has been approved for the treatment of several malignancies in the USA and was also one of the earlier agents to enter HCC clinical trials after sorafenib. Despite the encouraging results from phase II trials using single-agent bevacizumab, serious bleeding complications were reported in $11 \%$ of patients, and no phase III trials of single-agent bevacizumab have been performed. Cetuximab is a mAb targeting EGFR. In a phase II study of unresectable HCC treated with cetuximab alone, no significant antitumor activity was observed. Nevertheless, cetuximab in combination with gemcitabine and oxaliplatin (GEMOX) promoted progression-free survival. In addition, there are currently four mAbs against IGF-1R (cixutumumab, AVE1642, BIIB022 and IMC-A12), one $\mathrm{mAb}$ against TRAIL receptor 1 (mapatumumab) and one mAb against TRAIL receptor 2 (tigatuzumab) under investigation in phase I and II trials for patients with HCC.

There has been an increasing interest in blocking some enzymes that play critical roles in hepatocarcinogenesis and metastasis. Heparanase is an endoglycosidase that cleaves a component of tumor extracellular matrix heparan sulfate proteoglycans. This cleavage event results in the loss of basement membrane integrity and promotion of cell metastasis. As a 
powerful inhibitor of heparanase, PI-88 is currently under investigation in phase II/III trials of HCC patients. Nuclear factor-kB (NF-kB) is a proposed major target of proteasome inhibitors, which is critical for inflammatory cytokine expression in many cancers. Bortezomib, a proteasome inhibitor that can prevent the ubiquitination and proteolysis of its inhibitory partner, IkB, and then prohibit NF-kB transcriptional activity, is also in a phase III clinical trial [12].

In conclusion, though there are many molecular-targeted agents in clinical trials, only sorafenib has been reported to be effective in HCC treatment. However, owing to the modest benefit of overall survival and the possibility of increased metastases by sorafenib, new therapies to augment or replace sorafenib are urgently needed. Considering that no agent targeting a single pathway could achieve complete response in HCC, it is rational to simultaneously inhibit two or more different pathways in HCC therapy.

\section{Cancer Stem Cell-Targeted Therapies}

A wide range of studies in stem cell biology suggest that malignant tumors can be viewed as a hierarchy of heterogeneous cell populations with different biological properties, and that not all the cells in tumors have the ability to maintain tumor growth, but only a small subpopulation called cancer stem cells (CSCs). CSCs were first characterized in leukemia and thereafter have been identified in most tumor types. It has been well proven that CSCs are endowed with a self-renewal capability that gives rise to all the components of a heterogeneous tumor. Increasing evidence indicates that tumor development and relapse is closely associated with the propagation of CSCs. The CSC hypothesis has exerted a fundamental impact on our current perception of cancer therapy. We should now shift our attempts focusing on eliminating the bulk of rapidly proliferating and terminally differentiated cells to concomitantly targeting the minority CSCs. One potential reason for the limited benefits of the current cancer treatment is that treatments such as chemotherapy, radiotherapy and the majority of molecular-targeted therapies are directed against rapidly growing malignant cells and fail to eradicate quiescent CSCs, which are more resistant to these conventional modalities. Therefore, therapies that can target CSCs may hold promise for the improvement of the curative effects and the survival of cancer patients.

Substantial progress has been made to identify CSCs and understand their biological characteristics, providing the foundation for CSC-targeted therapies. To date, CSCs in HCC can be identified by several putative surface markers such as CD133, CD44, CD90, OV6, the epithelial cell adhesion molecular (EpCAM) and CD13, or by selecting the side population cells [13]. Moreover, a combination of multiple markers can be used for further selecting a population of cancer cells with stronger CSC characteristics.

One way to eliminate CSCs is to target these surface markers. Some studies investigated a murine antibody to human CD133 conjugated with monomethyl auristatin (a potent cytotoxic drug) to target CD133+ cells, a neutralizing antibody to human CD44 for blockage of CD44 in CD90+CD44+ cells, small interfering RNA to suppress the expression level of EpCAM, or CD13-neutralizing antibody or CD13 inhibitor ubenimex (bestatin) to block CD13 function; all of these strategies have shown remarkable antitumor effects both in vitro and in vivo [14].

The maintenance of stemness properties in CSCs and normal stem cells involves a diverse network of regulatory mechanisms. Several pathways have been identified to play important roles in the self-renewal capability of CSCs, including the WNT/ $\beta$-catenin, Notch and Hedgehog pathways, and thus present potential therapeutic targets for CSCs [15]. Furthermore, other signaling pathways such as transforming growth factor beta/SMAD, interleukin 6/STAT3 and 
extracellular signal-regulated kinase/MAPK pathways, and some genes such as PTEN, C-MYC and the polycomb gene BMI, may also be involved in the tumorigenicity of HCC CSCs. Indeed, blockade of the WNT pathway using mAbs or small molecule inhibitors targeting the components of this pathway can inhibit angiogenesis and tumor growth in vitro and in vivo. Suppression of the Notch pathway directed by mAbs targeting the Notch ligand Delta-like 4 or indirectly by inhibiting hypoxia-inducible factor 1 alpha has been shown to eradicate CSCs in mouse lymphoma and serially transplanted human acute myeloid leukemia in xenogeneic models. Some studied showed that Hedgehog inhibitors such as cyclopamine and small molecule inhibitors of Smo or Gli1/2 could also remarkably decrease cell viability and induce apoptosis in HCC cells [16]. In addition, emerging evidence also suggests an important role of miRNAs in regulating HCC CSCs [17]. Although research is still at an early stage, miRNA is also a potential target in HCC therapy, as some studies have reported an inhibitive effect of miRNAs on HCC CSCs through regulation of their expression.

Although CSC-targeted therapy sheds new light on HCC treatment, this strategy still has its disadvantages. First, none of the currently identified CSC markers or signaling pathways is specific for malignant cells. These markers or pathways also exist in normal stem cells or normal tissues. Thus, strategies targeting CSCs may inevitably cause damage to normal stem cells and influence liver regeneration. Second, we are not certain whether the cells selected by the markers are bona fide CSCs. Therefore, agreement is required on the phenotype of CSCs that can precisely identify HCC CSCs so as to not only discriminate CSCs from their counterparts but also from normal stem cells. Future research should investigate new agents that selectively and specifically target CSCs while leaving the normal stem cell population unaffected. It should also be noted that, to cure tumors such as HCC, both CSCs and non-CSCs should be eliminated since non-CSCs may convert to CSCs, and thus eradication of CSCs alone may not achieve complete regression of an established tumor. Therefore, therapies eliminating CSCs should be combined with conventional therapies targeting non-CSCs.

\section{Differentiation Therapy}

Differentiation therapies in oncology are broadly defined as strategies that induce malignant reversion. The idea that conversion of malignant cells to benign cells may be a possible therapeutic strategy against cancer in humans was first proposed by G. Barry Pierce in 1961, when differentiation was observed in teratocarcinoma cells. However, the mechanisms of self-differentiation of malignant cells were poorly understood. Therefore, the transformation of this unusual idea to a successful clinical practice was not realized until 1984 when the use of all-trans retinoic acid (ATRA) in the treatment of acute promyelocytic leukemia was reported. This breakthrough transformed this fatal disease into one of the most curable forms of leukemia [18].

Treating cancer by inducing differentiation is very attractive for physicians. Unlike conventional therapies such as cytotoxic chemotherapy and radiotherapy that directly eliminate tumor cells, this approach forces the differentiation of malignant cells into more mature cells that will then lose their malignant potential. In this case, less toxicity may be expected compared with conventional chemotherapies. Additionally, this strategy not only targets CSCs but also maturation-arrested proliferating cells. Unfortunately, unlike the situation of acute promyelocytic leukemia, the development of differentiation therapy for solid tumors is currently far from satisfactory.

Differentiation inducers for malignant tumors can be generally divided into two categories. The first category includes non-specific agents that exert activity on a wide range of cell and tissue types and includes histone deacetylase inhibitors (HDACIs), retinoids, dimethyl 
sulfoxide, peroxisome proliferator-activated receptor (PPAR $\gamma$ ) agonist, butyric acid, vesnarinone and so on. For example, retinoids are typical reagents for the treatment for acute promyelocytic leukemia and can also induce differentiation in a large number of cell types, such as embryonic cells, teratocarcinomas, melanomas, osteosarcoma, chondrosarcoma and rhabdomyosarcoma. HDACIs can induce the differentiation of various solid tumors and sarcomas. Although the therapeutic effects of non-specific differentiation inducers may be non-significant, most can potentiate the effect of conventional chemotherapy or radiation therapy. Therefore, the combination of differentiation therapy with conventional treatments might be a potential second-line treatment in patients with advanced cancer. The second category includes agents that only exhibit activity against a limited range of cell and tissue types, and these specific differentiation inducers may have a remarkable ability to induce differentiation. It has been reported that nerve growth factor could effectively induce the neuronal differentiation of the neuroendocrine cell line PC12 [19].

Hepatocyte nuclear factor $4 \alpha(\mathrm{HNF} 4 \alpha)$, a liver-enriched transcription factor, belongs to the nuclear hormone receptor superfamily. As a key member of the HNF family, HNF $4 \alpha$ is indispensable for hepatic epithelium formation during embryonic development and for epithelial phenotype maintenance of hepatocytes in mature liver. Substantial evidence has demonstrated that loss of HNF $4 \alpha$ expression is an important determinant of HCC progression, and transient inhibition of HNF $4 \alpha$ could initiate hepatocellular transformation [20]. Inspired by the success of the treatment of acute promyelocytic leukemia using ATRA, we have recently explored the possibility of HNF4 $\alpha$ differentiation therapy for HCC. Interestingly, our study clearly demonstrated that forced expression of HNF4 $\alpha$ could induce the differentiation of both non-CSCs and CSCs of HCC into hepatocyte-like cells both in vitro and in vivo. Intratumoral injection of HNF4 $\alpha$ displayed significant antitumor effects on transplanted tumor models. Striking suppressive effects on metastatic tumor formation were also achieved with systemic administration of HNF4 $\alpha$ [21]. Moreover, we also found that HNF4 $\alpha$ introduction could not only attenuate liver fibrosis in two distinct rat models, but also completely blocked hepatocarcinogenesis in a diethylnitrosamine-induced rat model [22, 23]. The inhibitory effect of HNF4 $\alpha$ on HCC development might be attributed to the suppression of hepatocyte epithelial-mesenchymal transition and the reduction of CSC generation through inhibition of the $\beta$-catenin signaling pathway. As the vast majority of HCC occurs in cirrhotic liver, the dual therapeutic effect of HNF $4 \alpha$ on fibrosis and HCC would be highly applicable in clinical practice. More recently, we also clarified that the miR-379-656 cluster plays an important role in the inhibitory effect of HNF $4 \alpha$ on HCC, suggesting that regulation of the HNF $4 \alpha$-miRNA cascade may have beneficial effects in the treatment of HCC [24, 25].

HNF1 $\alpha$ is another critical transcription factor of the HNF family and also plays a critical role in hepatocyte differentiation. Our results also showed that systemic administration of HNF1 $\alpha$ could eradicate orthotopic liver HCC nodules in NOD/SCID mice by inducing differentiation of hepatoma cells into mature hepatocytes [26].

It has been well documented that pluripotent stem cells can be induced from human somatic cells by defined transcription factors, implying that we might target the cell differentiation direction by appropriate factors. Thus, the more potent effect on carcinogenesis, progression and metastasis of HCC by HNF $4 \alpha$ compared with other known differentiation inducers may be attributed to the fact that HNF $4 \alpha$ acts as a transcription factor that is critical for hepatocyte differentiation. We believe that this HNF-based differentiation therapy might reveal new information for HCC in the near future. More generally, our results provide new clues for therapies of other malignant tumor types in that differentiation therapy may be achieved using corresponding differentiation-determining transcription factors. 


\section{Conclusions}

The treatment of patients with advanced HCC is still highly challenging. In the past decades, the in-depth elucidation of the molecular mechanisms of HCC has led to the identification of several potential molecular targets in HCC, and the development of moleculartargeted agents for the treatment of HCC has begun in earnest. CSC-based therapy and differentiation therapies have also shed new light on HCC treatment. Although the bench-to-bedside translational approach is still in its infancy, continued advances in our understanding of HCC will lead to the development of truly effective agents for the chemoprevention and treatment of HCC.

\section{References}

1 Siegel R, Naishadham D, Jemal A: Cancer statistics, 2012. CA Cancer J Clin 2012;62:10-29.

$\rightarrow 2$ European Association for the Study of the Liver; European Organisation for Research and Treatment of Cancer: EASL-EORTC clinical practice guidelines: management of hepatocellular carcinoma. J Hepatol 2012; 56:908-943.

-3 Verslype C, Rosmorduc 0, Rougier P; ESMO Guidelines Working Group: Hepatocellular carcinoma: ESMOESDO Clinical Practice Guidelines for diagnosis, treatment and follow-up. Ann Oncol 2012;23(suppl 7):vii41vii48.

4 Sangro B, Carpanese L, Cianni R, Golfieri R, Gasparini D, Ezziddin S, Paprottka PM, Fiore F, Van Buskirk M, Bilbao JI, Ettorre GM, Salvatori R, Giampalma E, Geatti O, Wilhelm K, Hoffmann RT, Izzo F, Iñarrairaegui M, Maini CL, Urigo C, Cappelli A, Vit A, Ahmadzadehfar H, Jakobs TF, Lastoria S; European Network on Radioembolization with Yttrium-90 Resin Microspheres (ENRY): Survival after yttrium-90 resin microsphere radioembolization of hepatocellular carcinoma across Barcelona clinic liver cancer stages: a European evaluation. Hepatology 2011;54:868-878.

5 Bruix J, Sherman M; American Association for the Study of Liver Diseases: Management of hepatocellular carcinoma: an update. Hepatology 2011;53:1020-1022.

-6 Wilhelm S, Carter C, Lynch M, Lowinger T, Dumas J, Smith RA, Schwartz B, Simantov R, Kelley S: Discovery and development of sorafenib: a multikinase inhibitor for treating cancer. Nat Rev Drug Discov 2006;5:835-844.

7 Ibrahim N, Yu Y, Walsh WR, Yang JL: Molecular targeted therapies for cancer: sorafenib mono-therapy and its combination with other therapies. Oncol Rep 2012;27:1303-1311.

-8 Zhang W, Sun HC, Wang WQ, Zhang QB, Zhuang PY, Xiong YQ, Zhu XD, Xu HX, Kong LQ, Wu WZ, Wang L, Song TQ, Li Q, Tang ZY: Sorafenib down-regulates expression of HTATIP2 to promote invasiveness and metastasis of orthotopic hepatocellular carcinoma tumors in mice. Gastroenterology 2012;143:1641-1649.

-9 Zhang QB, Sun HC, Zhang KZ, Jia QA, Bu Y, Wang M, Chai ZT, Zhang QB, Wang WQ, Kong LQ, Zhu XD, Lu L, Wu WZ, Wang L, Tang ZY: Suppression of natural killer cells by sorafenib contributes to prometastatic effects in hepatocellular carcinoma. PLoS One 2013;8:e55945.

10 Reichert JM, Rosensweig CJ, Faden LB, Dewitz MC: Monoclonal antibody successes in the clinic. Nat Biotechnol 2005;23:1073-1078.

11 Dawood S, Broglio K, Buzdar AU, Hortobagyi GN, Giordano SH: Prognosis of women with metastatic breast cancer by HER2 status and trastuzumab treatment: an institutional-based review. J Clin Oncol 2010;28:92-98.

12 Hamed O, Kimchi ET, Sehmbey M, Gusani NJ, Kaifi JT, Staveley-O'Carroll K: Impact of genetic targets on cancer therapy: hepatocellular cancer. Adv Exp Med Biol 2013;779:67-90.

13 Pang RW, Poon RT: Cancer stem cell as a potential therapeutic target in hepatocellular carcinoma. Curr Cancer Drug Targets 2012;12:1081-1094.

14 Yang ZF, Ho DW, Ng MN, Lau CK, Yu WC, Ngai P, Chu PW, Lam CT, Poon RT, Fan ST: Significance of CD90+ cancer stem cells in human liver cancer. Cancer Cell 2008;13:153-166.

15 Lee TK, Castilho A, Ma S, Ng IO: Liver cancer stem cells: implications for a new therapeutic target. Liver Int 2009;29:955-965.

16 Low JA, de Sauvage FJ: Clinical experience with Hedgehog pathway inhibitors. J Clin Oncol 2010;28:53215326.

17 Meng F, Glaser SS, Francis H, DeMorrow S, Han Y, Passarini JD, Stokes A, Cleary JP, Liu X, Venter J, Kumar P, Priester S, Hubble L, Staloch D, Sharma J, Liu CG, Alpini G: Functional analysis of microRNAs in human hepatocellular cancer stem cells. J Cell Mol Med 2012;16:160-173.

18 Ohno R, Asou N, Ohnishi K: Treatment of acute promyelocytic leukemia: strategy toward further increase of cure rate. Leukemia 2003;17:1454-1463.

19 Vaudry D, Stork PJ, Lazarovici P, Eiden LE: Signaling pathways for PC12 cell differentiation: making the right connections. Science 2002;296:1648-1649. 
-20 Hatziapostolou M, Polytarchou C, Aggelidou E, Drakaki A, Poultsides GA, Jaeger SA, Ogata H, Karin M, Struhl K, Hadzopoulou-Cladaras M, Iliopoulos D: An HNF4 $\alpha$-miRNA inflammatory feedback circuit regulates hepatocellular oncogenesis. Cell 2011;147:1233-1247.

21 Yin C, Lin Y, Zhang X, Chen YX, Zeng X, Yue HY, Hou JL, Deng X, Zhang JP, Han ZG, Xie WF: Differentiation therapy of hepatocellular carcinoma in mice with recombinant adenovirus carrying hepatocyte nuclear factor-4alpha gene. Hepatology 2008;48:1528-1539.

22 Yue HY, Yin C, Hou JL, Zeng X, Chen YX, Zhong W, Hu PF, Deng X, Tan YX, Zhang JP, Ning BF, Shi J, Zhang X, Wang HY, Lin Y, Xie WF: Hepatocyte nuclear factor 4alpha attenuates hepatic fibrosis in rats. Gut 2010;59:236-246.

-23 Ning BF, Ding J, Yin C, Zhong W, Wu K, Zeng X, Yang W, Chen YX, Zhang JP, Zhang X, Wang HY, Xie WF: Hepatocyte nuclear factor 4 alpha suppresses the development of hepatocellular carcinoma. Cancer Res 2010;70: 7640-7651.

24 Yin C, Wang PQ, Xu WP, Yang Y, Zhang Q, Ning BF, Zhang PP, Zhou WP, Xie WF, Chen WS, Zhang X: Hepatocyte nuclear factor- $4 \alpha$ reverses malignancy of hepatocellular carcinoma through regulating miR-134 in the DLK1DIO3 region. Hepatology 2013;58:1964-1976.

25 Xu WP, Yi M, Li QQ, Zhou WP, Cong WM, Yang Y, Ning BF, Yin C, Huang ZW, Wang J, Qian H, Jiang CF, Chen YX, Xia CY, Wang HY, Zhang X, Xie WF: Perturbation of miR-370-LIN28A-NF-kB regulatory circuit contributes to the development of hepatocellular carcinoma. Hepatology 2013;58:1977-1991.

-26 Zeng X, Lin Y, Yin C, Zhang X, Ning BF, Zhang Q, Zhang JP, Qiu L, Qin XR, Chen YX, Xie WF: Recombinant adenovirus carrying the hepatocyte nuclear factor-1alpha gene inhibits hepatocellular carcinoma xenograft growth in mice. Hepatology 2011;54:2036-2047. 